\title{
Axiomatic design study for automatic ship-to-ship mooring system for container operations in open sea
}

\author{
Yong Yook Kim, Kook-Jin Choi, Hyun Chung* and Phill-Seung Lee \\ Division of Ocean Systems Engineering, KAIST, Korea \\ (Received April, 20, 2011, Accepted June 8, 2011)
}

\begin{abstract}
To provide more rational design solutions at conceptual design level, axiomatic design method has been applied to solve critical part of a new engineering problem called Mobile Harbor. In the implementation, the Mobile Harbor, a functional harbor system that consists of a vessel with container crane approaches to a container ship anchored in the open sea and establishes a secure mooring between the two vessels to carry out loading and unloading of containers. For this moving harbor system to be able to operate successfully, a reliable and safe strategy to moor and maintain constant distance between the two vessels in winds and waves is required. The design process of automatic ship-to-ship mooring system to satisfy the requirements of establishing and maintaining secure mooring has been managed using axiomatic design principles. Properly defining and disseminating Functional Requirements, clarifying interface requirements between its subsystems, and identifying potential conflict, i.e. functional coupling, at the earliest stage of design as much as possible are all part of what need to be managed in a system design project. In this paper, we discuss the automatic docking system design project under the umbrella of KAIST mobile harbor project to illustrate how the Axiomatic Design process can facilitate design projects for a large and complex engineering system. The solidified design is presented as a result.
\end{abstract}

Keywords: mobile harbor, automatic ship-to-ship mooring, axiomatic design, vacuum suction pad, winch.

\section{Introduction}

As global economy grows ever more in its scale, there have increased sharp demand for global shipment. To cope with this demand, larger containerships that many existing ports are unable to accommodate are being introduced into the major stream of the global maritime logistics operation. On the other hand, there occur frequent needs for quick and economical distribution of goods to the places where there does not exist infrastructure to handle container cargoes to and from large container ships due to disasters, environmental reasons, or slow development of infrastructure in the region. To answer these kinds of unmatched demands, a new concept of container handling system, called Mobile Harbor, has been introduced and being developed in KAIST, Korea.

The Mobile Harbor is a "functional" harbor that consists of a vessel with a container handling crane and can move out to a large container ship anchored in an anchorage area for loading and unloading of the containers and return to a shallow or crowded harbor for inland transportation. In achieving this goal, diverse range of technologies have to be integrated together to solve many

*Corresponding author, Professor, E-mail: hyunny92@kaist.edu 
technical obstacles that has not been solved before. One of the major technical obstacles is quickly establishing secure and stable mooring between the container ship and the mobile harbor for crane operations when there are constant disturbances from waves, currents, and winds. Since the mobile harbor is designed to be about $250 \mathrm{TEU}$ and its length overall is about 80 meters, and the target container ships is about 15,000 TEU and the length overall is more than 380 meters, the Mobile Harbor only can cover few containers bays at the same time, thus it should be able to moor side-byside at any location along the port and starboard sides of the container ships.

In developing a novel system that has not existed previously, the principle of axiomatic design is an efficient design tool to clarify the Functional Requirements (FRs) of the complex system and corresponding Design Parameters (DP). The Axiomatic Design (AD) concept has been developed to fulfill various demands in engineering design by Suh (2001) and have applied to many successful engineering design processes (Hirschi 2002, Sarno et al. 2005, Suh 1998, Ullah 2005, Suh 2001).

In this study, the design process of establishing and maintaining secure mooring between a Mobile Harbor and a container ship has been managed using Axiomatic Design principles and all successfully applied to the concept design of automatic ship-to-ship mooring system.

\section{Axiomatic design}

For this kind of large and complex system design projects, the ability to effectively manage system concept design issues plays an essential role in determining the quality of outcomes. Properly defining and disseminating Functional Requirements, clarifying interface requirements between its subsystems, and identifying potential conflicts, i.e., functional coupling, at the earliest stage of design as much as possible are all part of what need to be managed in a system design project.

Axiomatic design concept has been developed to fulfill such various demands in engineering design by Suh (2001). The basic process of evolving a new design from scratch based on the axiomatic design principle is zigzagging process between defining the four different domains, which are customer needs or attributes (CAs), functional requirements (FRs), Design Parameters (DPs), and Process Variables (PVs) of the design and finding the mapping between them that can represent

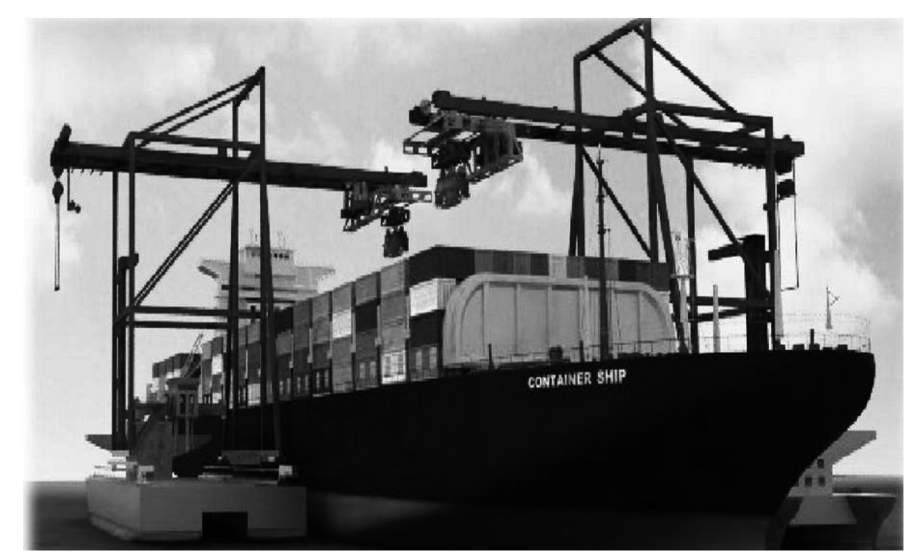

Fig. 1 Concept of Mobile Harbor operation 


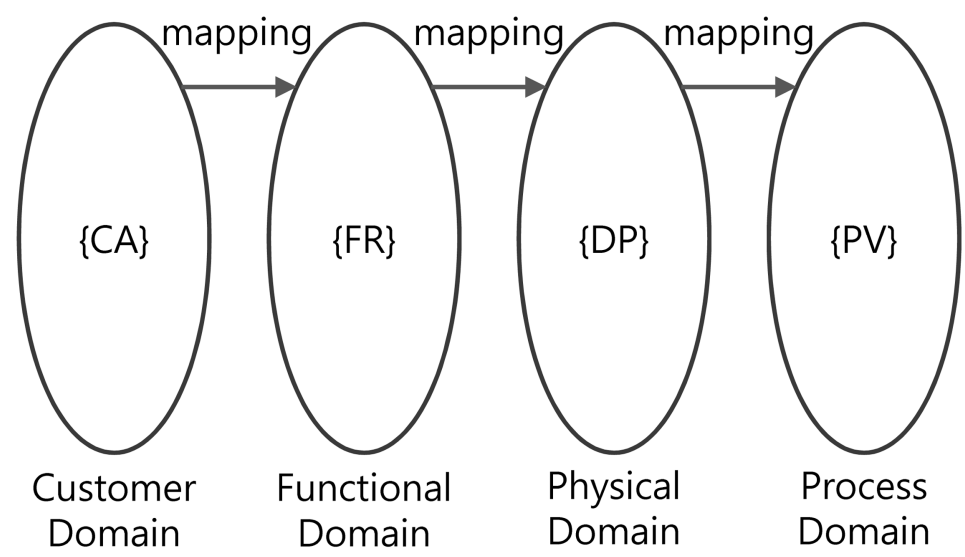

Fig. 2 Four domains of the design world

specific physical solutions. The design process should be handled with two basis axioms. One is the Independence Axiom and the other is the Information Axiom.

Independence Axiom: Maintain the independence of functional requirements Information Axiom: Minimize the information content of the design

The relationship between the FRs and the DPs can be represented as a design equation as shown below. The FRs and the DPs to be vectors and the relationship as a matrix.

$$
\left[\begin{array}{l}
F R 1 \\
F R 2 \\
F R 3
\end{array}\right]=\left[\begin{array}{ccc}
X & 0 & 0 \\
A_{21} & X & 0 \\
A_{31} & A_{32} & X
\end{array}\right]\left[\begin{array}{l}
D P 1 \\
D P 2 \\
D P 3
\end{array}\right]
$$

The matrix in the above equation is called the design matrix. To fulfill the Independence Axiom and result in uncoupled design, the design matrix should be diagonal or triangular. The details of the theory can be found in the references.

\section{Axiomatic study of automatic ship-to-ship mooring system}

The main functional requirement of Mobile Harbor ship-to-ship mooring system is establishing secure and safe mooring between the Mobile Harbor and the container ship so that cranes on the Mobile Harbor can perform loading and unloading containers to and from the container ship for required duration of operation. This main functional requirement of the docking system stems from the functional requirements of the whole Mobile Harbor system (Lee et al. 2010).

In the decomposition of main functional requirements of the Mobile Harbor, the functional requirements of the mooring system correspond to FR5: Moor to the container ship. This is because the top management of KAIST mobile harbor team decided to manage the sub-team based on the FRs of the Mobile Harbor system.

To moor one Mobile Harbor (MH) to a container ship, the process requires number of steps to 
accomplish. Firstly, the MH should approach to the appropriate docking area of the container ship while the container ship maintains a specific position. Once the MH is close to the container ship, the distance between the two vessels should be constantly maintained for a safe crane operation. Since the two vessels are close by, the two vessels could hit each other or make contacts. Therefore, any damage to the two vessels should not occur from these contacts. Finally, the mooring between the two vessels can only be established up to certain level of disturbances or sea state. So, the environment around the two vessels should be constantly checked and monitored so that the two vessels could be able to release mooring and establish certain distances from each other in time to avoid hazardous collision to each other. The list of these functional requirements and corresponding physically plausible design variables are listed as below.

\subsection{Decomposition of FR5}

FR5: Moor to the container ship

-FR51: Approach the MH to the docking location.

-FR52: Make the ship not to move away from an intended location.

-FR53: Maintain relative distance between the MH and the container ship.

-FR54: Minimize damage from a collision or contact between the MH and the container ship.

-FR55: Keep surrounding environment in check.

DP5: Docking and mooring system

-DP51: Azimuth thruster, bow thruster, and precise proximity maneuvering system.

-DP52: Anchor of container ship (one point mooring)

-DP53: Robot arm mooring system

-DP54: Adjustable fender system

-DP55: Operation condition (sea state and etc.) sensing / analysis system

Along the decomposition process, design matrix analysis is conducted to identify potential functional couplings. Since the design matrix is constructed in the middle of the design process where much design concepts is still being developed, many non-zero off-diagonal terms in the design matrix indicates potential interactions and conditions for those interactions as opposed to real couplings. The design matrix at the first level of the decomposition is shown below

$$
\left[\begin{array}{l}
F R 51 \\
F R 52 \\
F R 53 \\
F R 54 \\
F R 55
\end{array}\right]=\left[\begin{array}{lllll}
X & & & A_{1 .} & \\
& X & & & \\
& X & & \\
& A_{43} & X & \\
& & & & X
\end{array}\right]\left[\begin{array}{l}
D P 51 \\
D P 52 \\
D P 53 \\
D P 54 \\
D P 55
\end{array}\right]
$$

$\mathrm{A}_{\mathrm{ij}}$ are non-zero elements in the design matrix, and the rationale behind each of them is clarified.

- $\mathrm{A}_{14}$ : The size of the fender also affects the mooring distance.

- $\mathrm{A}_{43}$ : Since the robot arm mooring system directly attaches suction pad to the ship hull, the system can cause ship hull damage. 
One of the important properties of good design in axiomatic design is maintaining independence between each design axioms. However, in the case of the docking system, the coupling between certain design parameters and functional requirements were not being able to be avoided. The coupling terms are stated as below. Since FR51 and FR52 are about the design parameters of the container ships, we intentionally omitted the subsequent analysis of sub-system of them.

Among the DPs for FR5, the DP53, robot arm mooring system, can hardly define one specific simple physical entity. Therefore, the FR53 should be further decomposed and the decomposition is stated as below.

\subsection{Decomposition of FR53}

FR53: Maintain relative distance between the $\mathrm{MH}$ and the container ship.

-FR531: Attach the locking structure of the docking system to the outer hull of the container ship.

-FR532: Lock the docking system to the ship within certain distance.

-FR533: Lock the docking system for various surface conditions (ship hull curvature, welding bead, moisture, and others)

-FR534: Automatically subtract and contain/deploy, secure/release

-FR535: Limit relative movements and relative distances during mooring.

DP53: Robot arm mooring system

-DP531: Robot arm system

-DP532: Sensor system for relative movement and relative distance

-DP533: Vacuum suction pad system

-DP534: Robot arm control system

-DP535: Tension control winch mooring system (with mooring cables and fenders)

The design matrix is as follows

$$
\left[\begin{array}{l}
F R 531 \\
F R 532 \\
F R 533 \\
F R 534 \\
F R 535
\end{array}\right]=\left[\begin{array}{llllll}
X & & & \\
A_{21} & X & & & \\
& & & X & \\
A_{41} & A_{42} & A_{43} & X & \\
& A_{52} & & & X
\end{array}\right]\left[\begin{array}{l}
D P 531 \\
D P 532 \\
D P 533 \\
D P 534 \\
D P 535
\end{array}\right]
$$

The coupling terms are stated as below.

- $\mathrm{A}_{21}$ : The maximum distance for ship-to-ship mooring changes depending on the outreach distance of the robot arm.

- $\mathrm{A}_{41}$ : Construction of robot arm link affects robot arm subtract/deploy system

- $\mathrm{A}_{42}$ : Functions of relative movement and relative distance measurement sensors affect automatic operation functions of robot arm to subtract/deploy or lock/release operation.

- $\mathrm{A}_{43}$ : The shape and the weight of the vacuum suction pad affects automatic locking and releasing operation using robot arm

- $\mathrm{A}_{52}$ : Relative movement and relative distance measurement sensors are required to maintain relative movement and relative distance 
The DP531, robot arm system to attach the suction pad requires further decomposition to specifically indicate the detailed mechanism.

\subsection{Decomposition of FR531}

FR531: Attach the locking structure of the docking system to the outer plate of the container ship. -FR5311: When maintaining mooring state, partly carry or elude the mooring load

-FR5312: Dampen the forces from relative motion against the container ship

-FR5313: Find the location for attachment to compensate for draft changes

DP531: Robot arm system

-DP5311: Force-response joint or folding part

-DP5312: Damping device

-DP5313: Analysis system for vacuum suction pad attachment positioning

Constraints:

-C531: The system has to be designed to withstand additional forces from initial relative movement right after the attachment of suction pads

$$
\left[\begin{array}{l}
F R 5311 \\
F R 5312 \\
F R 5313
\end{array}\right]=\left[\begin{array}{lll}
X & & \\
& X & \\
& & X
\end{array}\right]\left[\begin{array}{l}
D P 5311 \\
D P 5312 \\
D P 5313
\end{array}\right]
$$

Determining whether proper distance and movement for locking of the system cannot be determined with one physical process and the FR532 can be decomposed as below.

\subsection{Decomposition of FR532}

FR532: Lock the docking system to the ship that approaches within certain distance

-FR5321: Senses whether attachable distance for vacuum suction pad using extendable robot arm has been reached

-FR5322: Extends robot arm so that the vacuum suction pad can be locked to the outer hull of the container ship

DP532: Relative movement and distance measurement sensor system

-DP5321: Distance sensor

-DP5322: Maximum outreach distance of the robot arm

Constraints:

- C531: Should maintain structural stability of the robot arms in the maximum outreach state

$$
\left[\begin{array}{l}
F R 5321 \\
F R 5322
\end{array}\right]=\left[\begin{array}{l}
X \\
X
\end{array}\right]\left[\begin{array}{l}
D P 5321 \\
D P 5322
\end{array}\right]
$$

For the system to attach to various surface conditions, the vacuum suction pad, DP533, can be 
used but this pad should full various functional requirements to result in more specific design.

\subsection{Decomposition of FR533}

FR533: Lock the docking system in various surface conditions (ship hull curvature, welding bead, moisture, and others)

-FR5331: Should be able to attach to uneven surfaces such as outer plates with weld beads for various surface conditions (moisture, rust, and other foreign particles)

-FR5332: Should be able to attach to the outer plate even when the surface of suctions pads and the surface of the outer plate are not parallel to each other

-FR5333: Should be able to attach to curved surface of the container ship

-FR5334: Should prevent slips from loads in tangential direction

DP533: Vacuum suction pad system

-DP5331: Rubber seal (shape and material)

-DP5332: Universal joint structure of the vacuum suction pad support part

-DP5333: Divided construction of vacuum suction pad

-DP5334: Shape of the rubber seals around the vacuum suction pad and the rubber supports inside of the vacuum suction pad

$$
\left[\begin{array}{l}
F R 5331 \\
F R 5332 \\
F R 5333 \\
F R 5334
\end{array}\right]=\left[\begin{array}{lllll}
X & & & \\
A_{21} & X & & \\
& A_{32} & X & 1 \\
& 1 & & X
\end{array}\right]\left[\begin{array}{c}
D P 5331 \\
D P 5332 \\
D P 5333 \\
D P 5334
\end{array}\right]
$$

- $\mathrm{A}_{21}$ : Make relative orientations between the suction pads and the outer plate of the container ship to be parallel to each other even when the surface of the suction pads and the surface of the outer plate are not approached to each other in parallel state by the cushioning effects of the rubber seals

- $\mathrm{A}_{32}$ : Makes divided suction pads to conform better to the curved surface by the action of the universal joint in the support part of the suction pad

The automatic function of the robotic arm should also satisfy various functional requirements to result in more specific design.

\subsection{Decomposition of FR534}

FR534: Automatically subtract and contain/deploy, lock/release

-FR5341: Make the suctions pads close to the outer plate of the container ship

-FR5342: Minimize the relative motions between the adjacent suction pads and the outer plate of the container ship

-FR5343: Automatically subtract and contain

-FR5344: Subtracts suction pads away from the container ship up to a certain distance right upon release

-FR5345: Orients the suction pads to be as parallel as possible to the outer plate 
-FR5346: Secure the suction pads to the outer plate of the container ship by forcing the suction pad close to the outer plate

\section{DP534: Robot arm control system}

-DP5341: Controller for lengthening direction joint (position control)

-DP5342: Controller for winch (for up and down movement)

-DP5343: Controller for slewing rotation (position control)

-DP5344: Force controller for lengthening direction joint (force control, spring-damper device)

-DP5345: Force controller for link parts of suction pads (force control, spring-damper device)

-DP5346: Force controller for slewing rotation (spring-damper device)

$$
\left[\begin{array}{l}
F R 5341 \\
F R 5342 \\
F R 5343 \\
F R 5344 \\
F R 5345 \\
F R 5346
\end{array}\right]=\left[\begin{array}{lllllll}
X & & & & & \\
0 & X & A_{23} & & & \\
A_{31} & A_{32} & X & & & \\
A_{41} & & & X & & \\
& & & & X & \\
A_{61} & & & & A_{64} & 0 & X
\end{array}\right]\left[\begin{array}{c}
D P 5341 \\
D P 5342 \\
D P 5343 \\
D P 5344 \\
D P 5345 \\
D P 5346
\end{array}\right]
$$

- $\mathrm{A}_{23}$ : Requires controller for slewing rotation to control the location of suction pads relative to the container ship in longitudinal direction

- $\mathrm{A}_{31}, \mathrm{~A}_{32}$ : Utilizes controllers for longitudinal and vertical directions to automatically subtract and contain the robot arms and to deploy the robot arms for operations

- $\mathrm{A}_{42}$ : Needs to retract the robot arms using position controller upon suction release

- $A_{61}$ : Needs to push the pads against the outer plate of the container ship for vacuum suction attachment

- $\mathrm{A}_{64}$ : The settings of force controller influences the scale of the pressing force

Limiting relative moments to certain degree also requires number of sub processes.

\subsection{Decomposition of FR535}

FR535: Limits relative movements to be under certain range during mooring

-FR5351: Limits relative surge to be under certain range

-FR5352: Limits relative sway to be under certain range

-FR5353: Limits relative yaw to be under certain range

-FR5354: Maintains the distance between the ships to be over certain minimum distance

-FR5355: Maintains the distance between the ships to be under certain maximum distance

DP535: Winch system with tension control (including mooring cable)

-DP5351: Mooring cables and winches arranged in diagonal directions

-DP5352: Even numbered mooring cables and winches symmetrically arranged in longitudinal direction

-DP5353: Multiple sets of mooring cables and winches, which are apart from each other in longitudinal direction 
-DP5354: Fender

-DP5355: Mooring cable

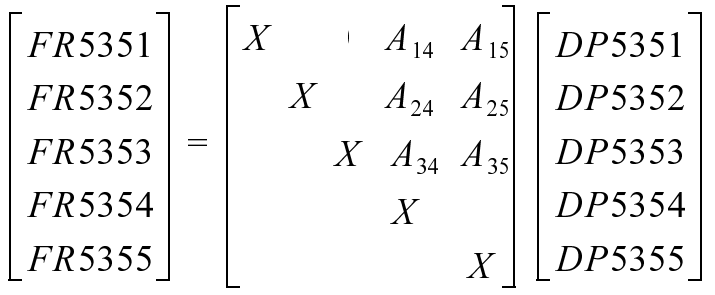

- $\mathrm{A}_{14}$ : Surge motion is affected by friction forces occurring from fenders

- $\mathrm{A}_{24}$ : Relative sway movements are affected by the size, stiffness, and damping of the fender

- $\mathrm{A}_{15}, \mathrm{~A}_{25}, \mathrm{~A}_{35}$ : Surge, sway, and yaw movements are affected by the stiffness of mooring cables

- $\mathrm{A}_{34}$ : Yaw motion is affected by the arrangements of fenders in the longitudinal direction

To minimize damage from the collision between the two vessels, fender can be used but is required to fulfill various decomposed functional requirements to reach to more specific design choice. The conceptual arrangement of the docking system is shown in Fig. 3.

\subsection{Decomposition of FR54}

FR54: Minimize damage from the collision between the MH and the container ship. -FR541: Absorb impact energy during berthing -FR542: Maintain minimum relative distance between the MH and the container ship -FR543: Prevent damages from relative movements between the MH and the container ship

DP54: Adjustable fender system

-DP541: Energy absorption quality of the fender (size and quality of the material)

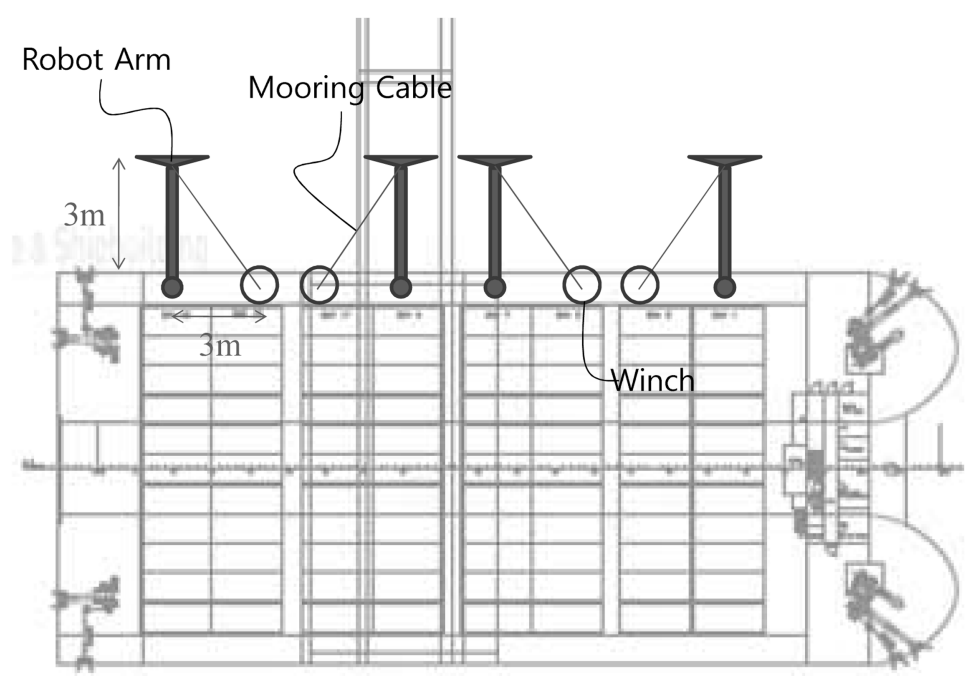

Fig. 3 Arrangement of robot arms and winches for docking system 
-DP542: Adjustable support structure

-DP543: Arrangement of main and supplementary fenders

$$
\left[\begin{array}{l}
F R 541 \\
F R 542 \\
F R 543
\end{array}\right]=\left[\begin{array}{ll}
X & \\
A_{21} & X \\
A_{31} & A_{32} X
\end{array}\right]\left[\begin{array}{l}
D P 541 \\
D P 542 \\
D P 543
\end{array}\right]
$$

- $\mathrm{A}_{21}$ : The size of the main fender affects the minimum distance between the ships.

- $\mathrm{A}_{31}$ : The energy absorption capacity of the fender affects the capability of the fender to minimize the damage from impact

The arrangement of fenders, DP543, also requires fulfilling various functional requirements.

\subsection{Decomposition of FR543}

FR543: Prevent any possible damage from relative movements between the $\mathrm{MH}$ and the container ship

-FR5431: Prevent damages from relative roll motion

-FR5432: Prevent damages from relative yaw motion

-FR5433: Prevent damages from relative sway motion

DP543: Arrangement of main and supplementary fenders

-DP5431: Supplementary fender (vertical position and size)

-DP5432: Multiple main fenders apart from each other in longitudinal direction

-DP5433: Multiple main fenders symmetrically arranged in longitudinal direction

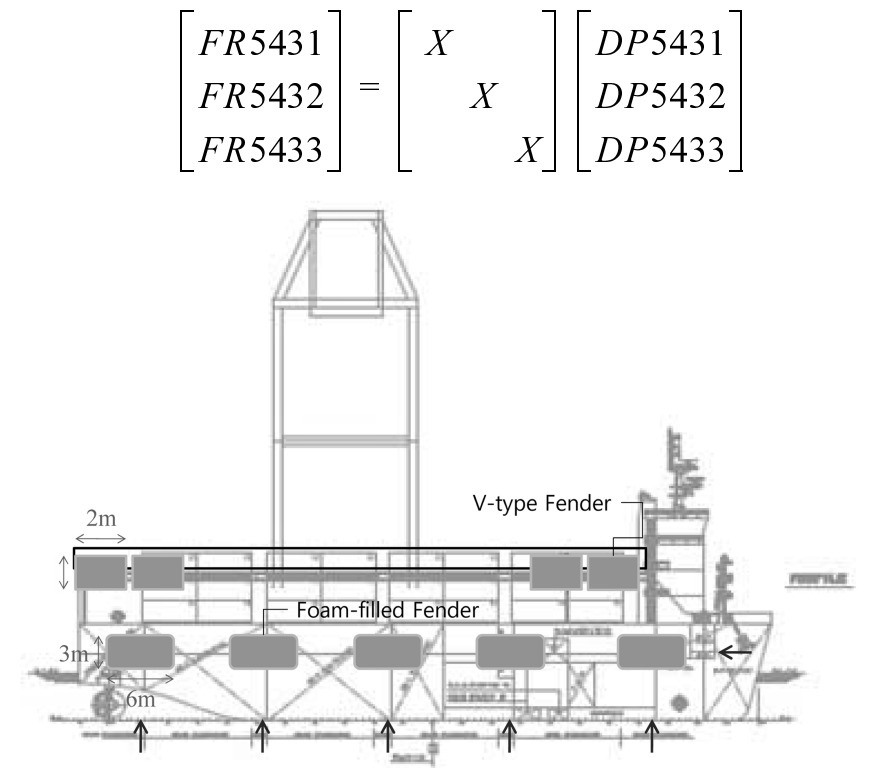

Fig. 4 Arrangement of fenders to avoid collision in case of excessive rolling and yawing 
According to the detailed functional requirements the fenders should be arranged as shown in Fig. 4.

To guarantee the safety of the Mobile Harbor and the docking system the surrounding environment should be closely monitored fulfilling various emergency situations that can be decomposed as below.

\subsection{Decomposition of FR55}

FR55: Monitor surrounding environment.

-FR551: Sense emergency situations of ship itself

-FR552: Sense the occurrence of excessive relative motions between ships occur

-FR553: Sense excessive external load

-FR554: Sense vacuum pressure change

-FR555: Sense abnormal change mooring cable tension

-FR556: Quick release of docking system in the case of abnormal situation

DP55: Operation condition (sea state and etc.) measurement / analysis system

-DP551: Communications with higher level system

-DP552: Sensor for relative motion and distance

-DP553: Load sensor for robot arms

-DP554: Vacuum pressure monitoring system

-DP555: Tension control response monitoring system

-DP556: Manual switch for forced release

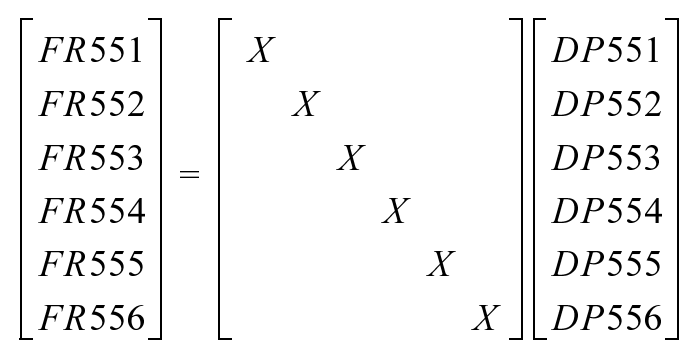

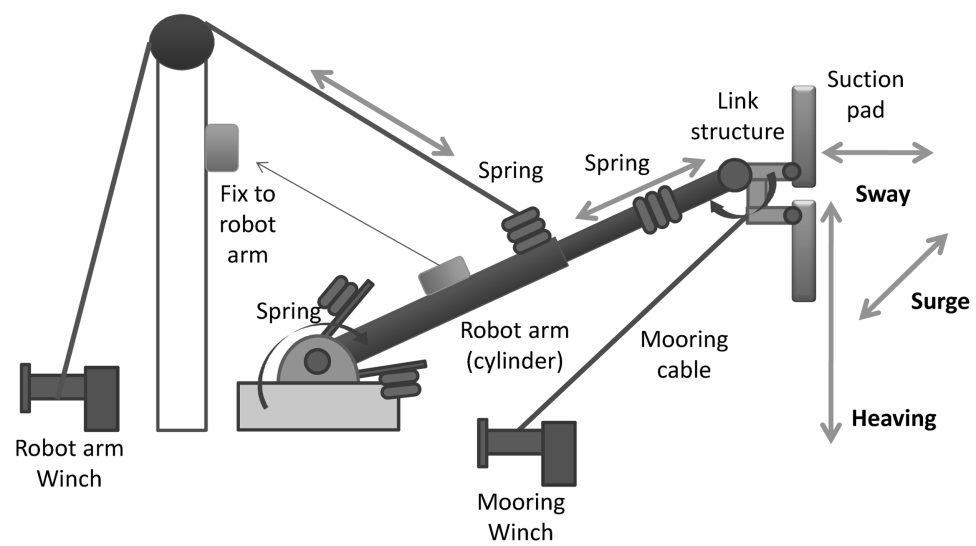

Fig. 5 Conceptual design of the Mobile Harbor automatic docking system based on the axiomatic design study 


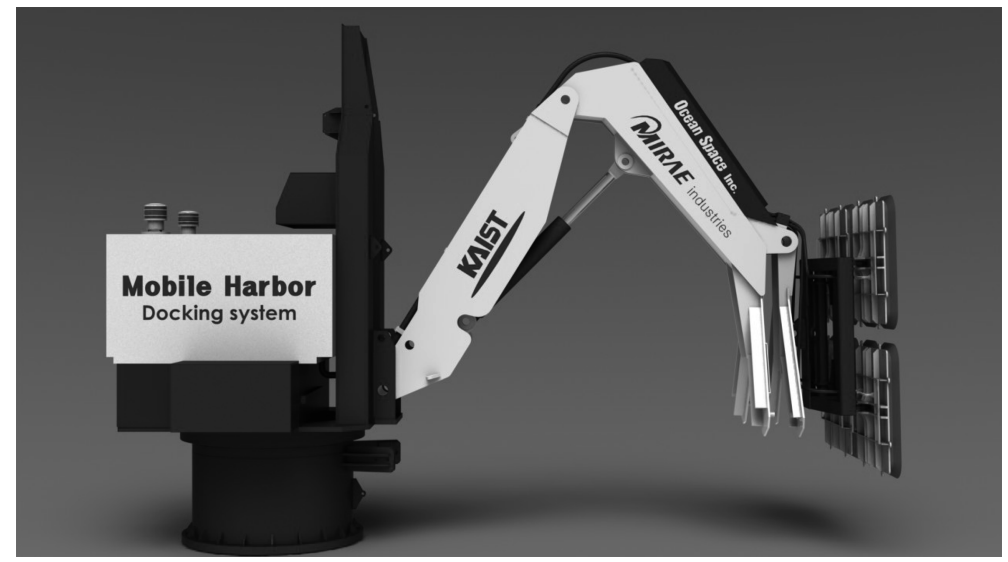

Fig. 6 Finalized design based on the concept design

The resulting conceptual design to fulfill all the functional requirements is shown in Fig. 5. Based on the concept design, the basic design has been determined including manufacturing considerations, which is shown in Fig. 6 . The basic design is developed via close interaction with manufacturer and solidified in about $3+$ months. All the concept design's characteristics are captures but some of the features are replaced with functionally equivalent off-the-shelf components for economical purpose. The system is manufactured and tested in real sea condition and we conclude that the concept design is a success, effectively satisfying all the functional requirements and validating its functionality in real sea conditions, as shown in Fig. 7.

\section{Conclusions}

The conceptual design of a novel automatic ship-to-ship mooring system has been found based on

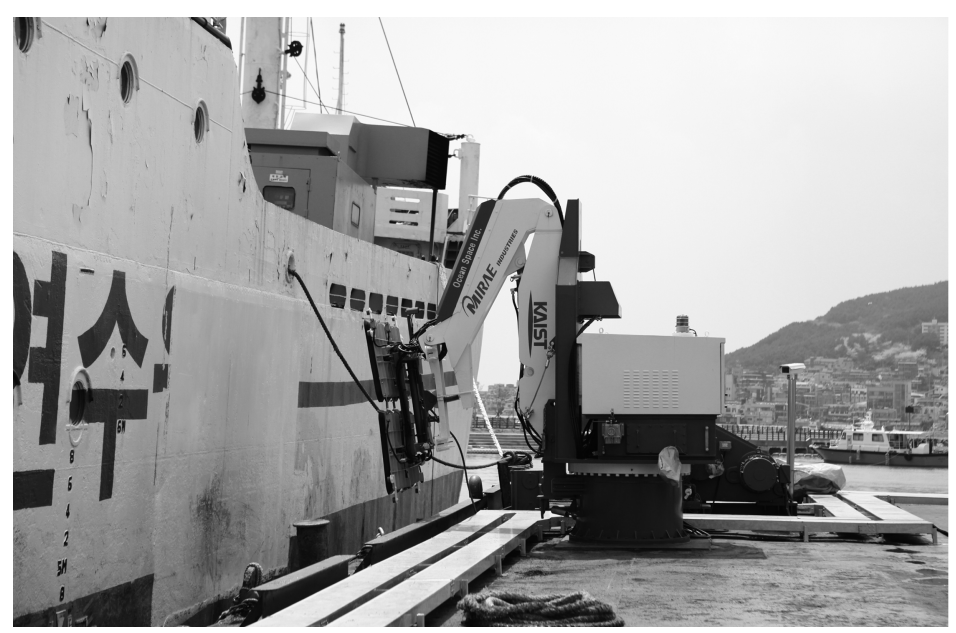

Fig. 7 Sea trial of the automatic ship-to-ship mooring system 
axiomatic design strategy. The axiomatic design process offers a suitable framework for the system concept development and design project. It provides a framework where diverse system design tasks, from customer needs to system interface identification, and to the physical system design, can be effectively planned and managed. Explicitly starting FRs for each sub-system help hierarchical decomposition of design requirements, and associating FRs with relevant DPs dismisses ambiguity of system boundary for each sub-system and thereby establishing clear functionality of the system. Design matrix analysis identifies all system functional coupling issues and ensures design decision made for different sub-subsystem to be in accord with each other and does not conflict to each other. Thus, more reasonable and realistic conceptual design of new engineering system has been found in earlier design stage. Also effectively conveying these information obtained by systems design and integration/engineering activities during the long design process is another critical aspect.

\section{Acknowledgments}

This work was supported by the Technology Innovation Program (10036234, Development of automatic ship-to-ship docking system) funded by the Ministry of Knowledge Economy (MKE, Korea)

\section{References}

Hirschi, N.W., and Frey in, D.D (2002), "Cognition and complexity: An experiment on the effect of coupling in parameter design," Research in Engineering Design-Theory Applications and Concurrent Engineering, 13(3), 123-131.

KAIST, 2009 Mobile Harbor Fundamental Technology Development Research Project Report, 2010.

Lee, T. and Park, G.J. (2010), "Managing System Design Process Using Axiomatic Design: A Case on KAIST Mobile Harbor Project ", SAE Int. J. of Mater. Manuf., 3(1), 125-132.

Sarno, E., Kumar, V. and Li, W. (2005), "A hybrid methodology for enhancing reliability of large systems inconceptual design and its application to the design of a multiphase flow station", Research in EngineeringDesign, 16(1-2), 27-41.

Suh, N.P. (1998), "Axiomatic design theory for systems", Research in Engineering Design-Theory Applications and Concurrent Engineering, 10(4), 189-209.

Suh N.P. (2001), Axiomatic design: advances and applications. New York: Oxford University Press.

Terminal Capacities as a Competitive Factor: New Forecasts for Container Shipping, HypoVereinsbank, 2008.

Ullah, A. (2005), "Handling design perceptions: an axiomatic design perspective", Research in Engineering

Design, 16(3), 109-117. 